\title{
Avaliação nutricional de crianças do Vale do Alto Jequitinhonha com a utilização das novas curvas de crescimento do NCHS e da OMS
}

\author{
Nutritional assessment of children of the Jequitinhonha Valley region in Brazil with NCHS \\ and the new WHO growth charts
}

Francisco José F. Silveira ${ }^{1}$, Joel Alves Lamounier ${ }^{2}$

\section{RESUMO}

Objetivo: Analisar comparativamente a avaliação nutricional de crianças em uma região carente do estado de Minas Gerais, utilizando curva de crescimento padrão de referência do NCHS e da Organização Mundial da Saúde (OMS).

Métodos: Estudo transversal realizado em três municípios da região do Alto Jequitinhonha, Minas Gerais. Foram incluídas as crianças com até 24 meses de idade, residentes nas áreas rurais e urbanas. A coleta de dados foi feita em julho de 2000. Análises comparativas foram feitas utilizando-se as curvas de crescimento do NCHS e da OMS com os programas Epi-Info 6.04b, Excel e Anthro 2005.

Resultados: Foram analisados dados de 450 crianças. A população era predominantemente de baixo nível socioeconômico, sendo 85,3\% com renda per capita inferior a meio salário mínimo. Utilizando-se respectivamente as curvas da OMS e do NCHS, o déficit peso/altura foi detectado em 4,2 e 2,2\% em crianças com até cinco meses e 2,3 e 3,2\% com 12 ou mais meses; o déficit altura/idade em 18,8 e 7,5\% com até cinco meses e 2,8 e 7,4\% com 12 meses ou mais; o sobrepeso em 12,5 e 8,6\% com até cinco meses e 7,4 e 5,1\% com 12 meses ou mais.

Conclusões: Foram encontradas diferenças na detecção de deficiências nutricionais. As curvas da OMS são mais atualizadas e foram elaboradas a partir de uma amostra composta de crianças vivendo em boas condições ambientais e de hábitos alimentares saudáveis, o que possibilitou seu crescimento adequado, podendo ser consideradas mais apropriadas para o acompanhamento do crescimento.

Palavras-chave: avaliação nutricional; padrões de referência; Organização Mundial da Saúde.

\section{ABSTRACT}

Objective: To compare the nutritional assessment of children of low socio-economic status in a poor region of Minas Gerais, Brazil, using NCHS and World Health Organization (WHO) growth reference standards.

Methods: Cross-sectional study performed in three cities of the Alto Jequitinhonha Valley, Minas Gerais, Brazil. Data were collected during July, 2000. Comparative analysis was made between NCHS and the new WHO growth charts by Epi-Info 6.04b, Excel and Anthro 2005 programs.

Results: A total of 450 children were assessed in the study. The socioeconomic status of the children was low and $85.3 \%$ of their family earned less than half of the minimum wage per capita. Using respectively WHO and NCHS growth charts, weight/height deficiency was detected in 4.2 and $2.2 \%$ in children up to five months and 2.3 and $3.2 \%$ in those with 12 months or more; height/ age deficiency was noted in 18.8 and $7.5 \%$ of the children up to five months and 2.8 and $7.4 \%$ in those with 12 months or more; overweight was shown in 12.5 and $8.6 \%$ up to five months of age, 7.4 and $5.1 \%$ in children with 12 months or more.

Conclusions: Differences in the detection of nutritional deficits were found. The WHO growth chart is more updated and the data was collected from children in good environment conditions and healthy feeding habits, favoring their normal growth. The WHO chart may be considered more adequate to assess children growth.

Key-words: nutritional assessment; reference standards; World Health Organization.
Instituição: Faculdade de Ciências Médicas de Minas Gerais e Universidade Federal de Minas Gerais (UFMG), Belo Horizonte, MG, Brasil 'Doutor em Pediatria. Professor assistente da Faculdade de Ciências Médicas de Minas Gerais (FCMMG), Belo Horizonte, MG, Brasil

${ }^{2}$ Doutor em Pediatria. Professor Titular do Departamento de Pediatria da Faculdade de Medicina da UFMG, Belo Horizonte, MG, Brasil
Endereço para correspondência:

Francisco José Ferreira da Silveira

Rua Galba Veloso, 304, apto 404 - Santa Tereza

CEP 31015-080 - Belo Horizonte/MG

E-mail: saujr@uol.com.br

Recebido em: 3/6/08

Aprovado em: 2/11/08 


\section{Introdução}

As curvas de crescimento representam padrões de crescimento de crianças normais e constituem valioso instrumento de avaliação das condições de saúde da população infantil, principalmente quando aplicadas de modo adequado pelos profissionais de saúde. São elaboradas a partir de estudos com indivíduos considerados normais, supostamente em condições ambientais favoráveis ao desenvolvimento de seus potenciais de crescimento e desenvolvimento. As mais conhecidas e utilizadas no Brasil são as curvas de crianças americanas publicadas pelo National Center for Health Statistics (NCHS) ${ }^{(1)}$, recomendadas pela Organização mundial de Saúde (OMS), e as de Marcondes et al, elaboradas com crianças de Santo André( ${ }^{(2)}$. Mais recentemente, surgiram as curvas do Centers for Disease Control and Prevention (CDC) $)^{(3)}$ com dados de crescimento de crianças Americanas (CDC, 2000) e elaboradas a partir de levantamentos mais atuais em comparação ao padrão do NCHS. Os dados foram coletados de 1963 a 1994 nos Estados Unidos e o novo referencial foi publicado em maio de 2000, trazendo algumas novidades, como a introdução do índice de massa corpórea e a inclusão de vários grupos étnicos na amostra. No entanto, ainda as curvas do CDC não expressavam corretamente o crescimento de crianças amamentadas, principalmente em aleitamento materno exclusivo, já que os lactentes que fizeram parte da amostra combinavam alimentação com fórmulas e leite materno.

Em abril de 2006, a OMS apresentou as novas curvas de crescimento para crianças com até cinco anos de idade, construídas com base em um estudo populacional realizado de 1997 a 2003 em cinco países: Brasil, Gana, Índia, Omã e Estados Unidos. A pesquisa foi denominada Estudo multicêntrico de referência do crescimento (MGRS). Participaram apenas crianças amamentadas e que seguiram padrões de alimentação considerados satisfatórios atualmente, em especial, em relação ao aleitamento materno, e que viviam em condições ambientais propícias para crescimento normal $^{(4)}$. Dessa forma, as curvas resultantes representam um padrão de crianças com alimentação adequada, vivendo em boas condições ambientais, sendo uma referência internacional, visto que foram obtidas com dados coletados em vários países.

As curvas de crescimento são importantes para avaliar a situação nutricional de crianças, além de serem úteis na detecção de alterações da saúde infantil. A OMS considera as novas curvas mais adequadas do que aquelas utilizadas anteriormente, recomendando sua implementação no Brasil e em outros países.

A aplicabilidade das novas curvas já foi demonstrada em um estudo que incluiu quatro países de condições socioeconômicas diferentes ${ }^{(5)}$. No entanto, poucos estudos foram realizados com o intuito de avaliar as novas curvas e os resultados obtidos em comparação à utilização das curvas do NCHS. Dessa forma, o objetivo deste estudo foi analisar comparativamente a situação nutricional de crianças com até 24 meses de idade de uma região de baixo nível socioeconômico do estado de Minas Gerais, utilizando-se as curvas da OMS e do NCHS.

\section{Métodos}

Parte de um estudo transversal realizado nos municípios de Carbonita, São Gonçalo do Rio Preto e Datas, região do alto Jequitinhonha, em Minas Gerais, cujo objetivo primário foi avaliar o tempo de aleitamento materno e o estado nutricional de crianças residentes nos locais citados. Os três municípios pertencem ao Consórcio Intermunicipal de Saúde do Alto Jequitinhonha (Cisaje) e são subordinados à Diretoria Regional de Saúde de Diamantina, com localização respectivamente a 30, 50 e $120 \mathrm{~km}$ da cidade. Tais municípios foram incluídos no estudo por terem cobertura total do Programa de Saúde da Família (PSF) e por terem convênio para a realização de estágio curricular do Internato Rural da Faculdade de Ciências Médicas de Minas Gerais, facilitando a concretização da pesquisa. Os municípios de Carbonita, São Gonçalo do Rio Preto e Datas apresentam 11 mil, 3 mil e 5.100 habitantes, respectivamente. O poder aquisitivo da população é baixo nos três municípios, sendo as atividades econômicas principais a exploração madeireira e carvoarias em Carbonita e a agropecuária em São Gonçalo do Rio Preto e Datas.

Foram consideradas para o estudo todas as crianças com até 24 meses de idade nos três municípios. A coleta de dados foi realizada em julho de 2000 a partir de entrevistas com mães ou responsáveis pelas crianças feitas por alunos de Medicina da Universidade Federal de Minas Gerais (UFMG) e da Faculdade de Ciências Médicas de Minas Gerais (FCMMG). Os alunos foram treinados para a utilização dos questionários e obtenção dos dados antropométricos durante os dois meses anteriores ao estudo. Todo o trabalho de treinamento e coleta de dados foi feito mediante supervisão do investigador principal, com estudo piloto prévio dois meses antes do início da pesquisa. 
O questionário utilizado continha perguntas fechadas e permitia a coleta das seguintes variáveis: identificação, data de nascimento, data da entrevista, aleitamento materno, idade da interrupção da amamentação, dados socioeconômicos, peso e comprimento da criança. As crianças foram pesadas utilizando-se balanças pediátricas, com nível de precisão de $10 \mathrm{~g}$, e medidas em posição horizontal por meio de réguas antropométricas.

$\mathrm{Na}$ área urbana, a coleta de dados foi feita no Centro de Saúde e nas próprias residências das crianças, caso as mães não comparecessem. Nas áreas rurais, em virtude da dispersão dos domicílios, as entrevistas eram feitas em locais previamente escolhidos de acordo com as condições de cada localidade (postos de saúde, escolas, centros comunitários). Entretanto, o recrutamento das mães e crianças foi feito nos domicílios, com o auxílio dos agentes comunitários de saúde.

Os dados foram analisados com os programas Epi-Info versão 6.04b, Microsoft ${ }^{\circledR}$ Excel e Anthro 2005, da OMS. A classificação de desnutrição foi feita de acordo com as recomendações da OMS, definindo-se como desnutridas as crianças que estivessem abaixo de -2 escore $Z$ e desnutridas graves aquelas que estivessem abaixo de -3 escore $Z$ para cada indicador. Foram considerados os indicadores peso/ idade, altura/idade e peso/altura. Foram ainda consideradas com sobrepeso as crianças com indicador peso/altura maior de +2 escore $Z$.

Os resultados foram elaborados na forma de gráficos, considerando-se os padrões do NCHS e da OMS, de acordo com três faixas etárias (até cinco meses, seis a 11 meses, 12 a 24 meses) para a detecção de desnutrição e obesidade.

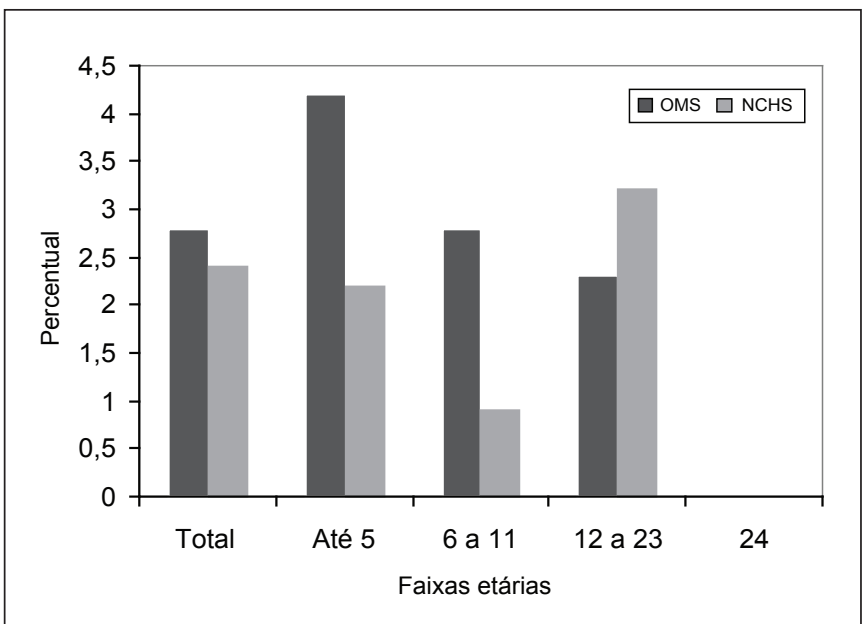

Figura 1 - Percentual de déficit peso/altura: curvas OMS e $\mathrm{NCHS}$.
Foram também feitas comparações das médias obtidas no estudo, considerando-se as duas curvas, referentes a sete faixas etárias (até três meses, quatro a sete meses, oito a 11 meses, 12 a 15 meses, 16 a 19 meses, 20 a 23 meses, 24 meses).

A pesquisa foi aprovada pelo Departamento de Pediatria e pelo Comitê de Ética em Pesquisa da UFMG.

\section{Resultados}

Foram analisadas 450 crianças com até 24 meses de idade, sendo 234 delas $(52,0 \%)$ do sexo masculino e 216 $(48,0 \%)$ do sexo feminino. Em relação à faixa etária, 21,8\% tinham menos de seis meses, $25,1 \%$ de seis a 11 meses, $24,4 \%$ de 12 a 17 meses e $28,7 \%$ de 18 a 24 meses. A população era predominantemente de baixa renda, sendo que $85,3 \%$ tinham renda per capita inferior a meio salário mínimo. Os hábitos alimentares eram inadequados, com baixa mediana de tempo de aleitamento materno exclusivo (1,5 meses), apesar do valor médio de 10,9 meses para o aleitamento materno.

Em relação à comparação entre as curvas do NCHS e da OMS na detecção de crianças desnutridas, observou-se déficit peso/altura em $4,2 \%$ das crianças com até cinco meses considerando-se as curvas da OMS e 2,2\% com as curvas do NCHS; de seis a 11 meses, respectivamente 2,8 e $0,9 \%$; com 12 meses ou mais, 2,3 e 3,2\% (Figura 1). Já o déficit altura/idade, foi encontrado em 18,8 e 7,5\% em crianças com até cinco meses; 17,9 e $14,0 \%$ de seis a 11 meses e 14,8 e $11,1 \%$ com 12 meses ou mais (Figura 2). O déficit peso/ idade foi encontrado em 9,4 e 8,6\% das crianças com até

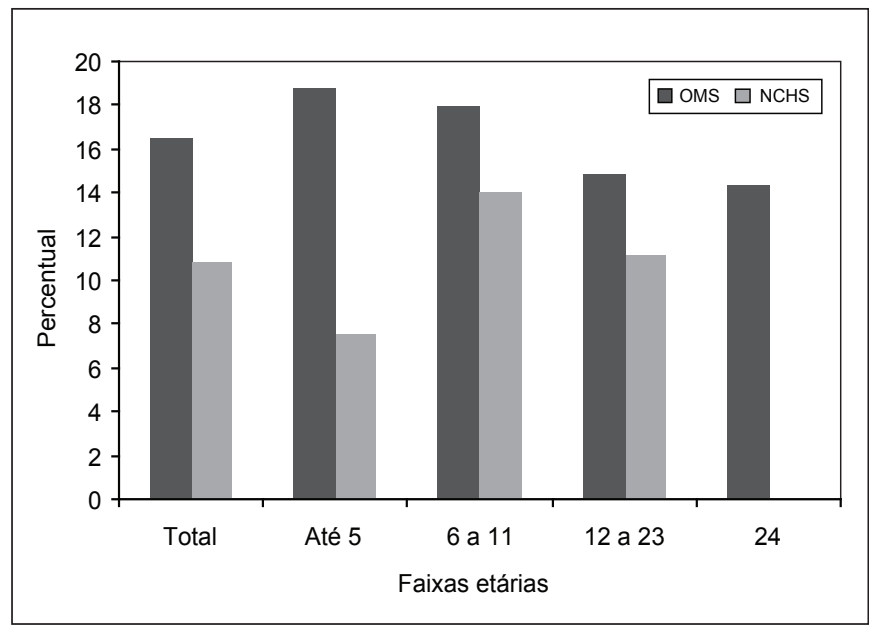

Figura 2 - Percentual de déficit altura/idade: curvas OMS e NCHS. 
cinco meses, 7,5 e 8,4\% de seis a 11 meses, 2,8 e 7,4\% com 12 meses ou mais (Figura 3). Para a detecção de sobrepeso, as curvas da OMS e do NCHS encontraram valores de 12,5 e $8,6 \%$, respectivamente, nas crianças de até cinco meses, 7,5 e $6,5 \%$ de seis a 11 meses e 7,4 e 5,1\% com 12 meses ou mais (Figura 4).

Em relação às médias dos valores de escore $Z$ a partir das duas curvas, para o indicador peso/idade verificou-se que os valores foram menores considerando-se o padrão da OMS nos primeiros meses, havendo uma intersecção no período de sete a oito meses; a partir de então, os valores foram menores para o padrão do NCHS (Figura 5). Já para o indicador altura/idade, os valores foram menores com o padrão da OMS nos primeiros meses, igualando-se entre sete e oito meses e tornando-se menores novamente a partir de 16 meses (Figura 6).

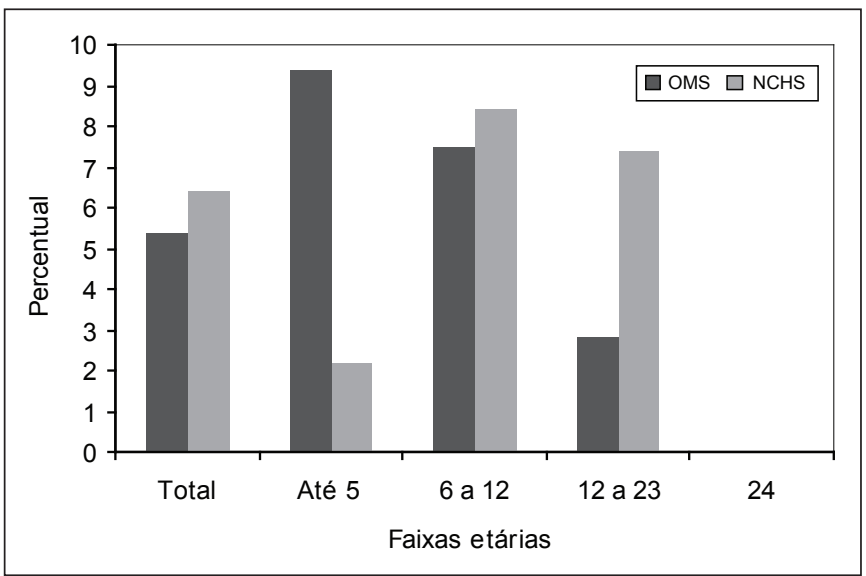

Figura 3 - Percentual de déficit peso/idade: curvas OMS e NCHS.

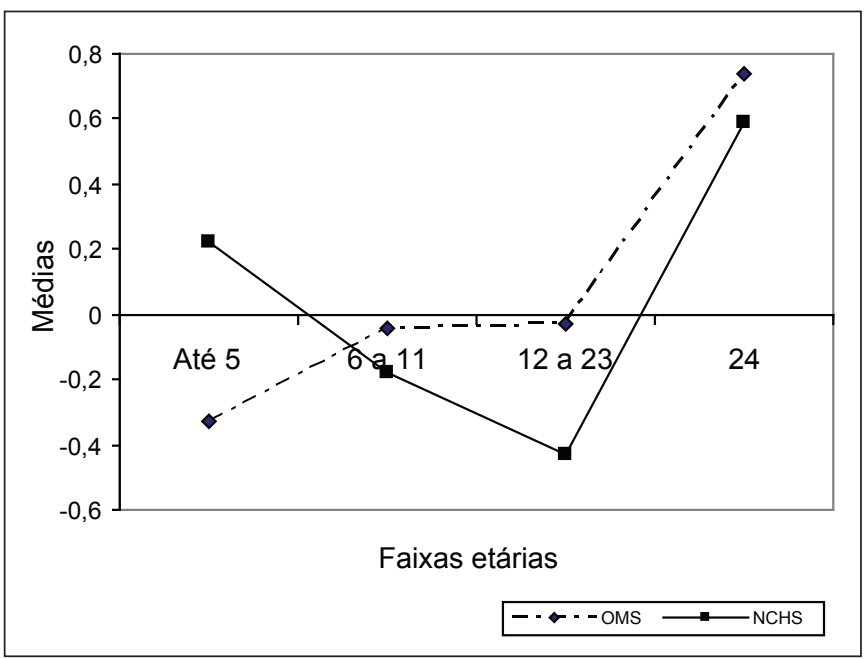

Figura 5 - Comparações de médias peso/idade: curvas OMS e NCHS.

\section{Discussão}

As curvas de crescimento do NCHS, que há algumas décadas são utilizadas em países de todo o mundo, inclusive por recomendação da OMS, têm sido alvo de questionamentos e suas deficiências já foram analisadas em várias publicações ${ }^{(6-9)}$. As curvas do NCHS são recomendadas para aplicação nos Estados Unidos desde 1977. Posteriormente, foram também recomendadas pela Organização Mundial de Saúde para uso internacional e foram adotadas pelo Ministério da Saúde no Brasil. Consideradas como padrão, tais curvas corresponderiam mais prontamente aos objetivos de avaliação de crescimento e estado nutricional de crianças em diferentes populações. As curvas do NCHS foram recomendadas devido às evidências de que o crescimento é influenciado por fatores como nutrição, ambiente, condições socioeconômicas

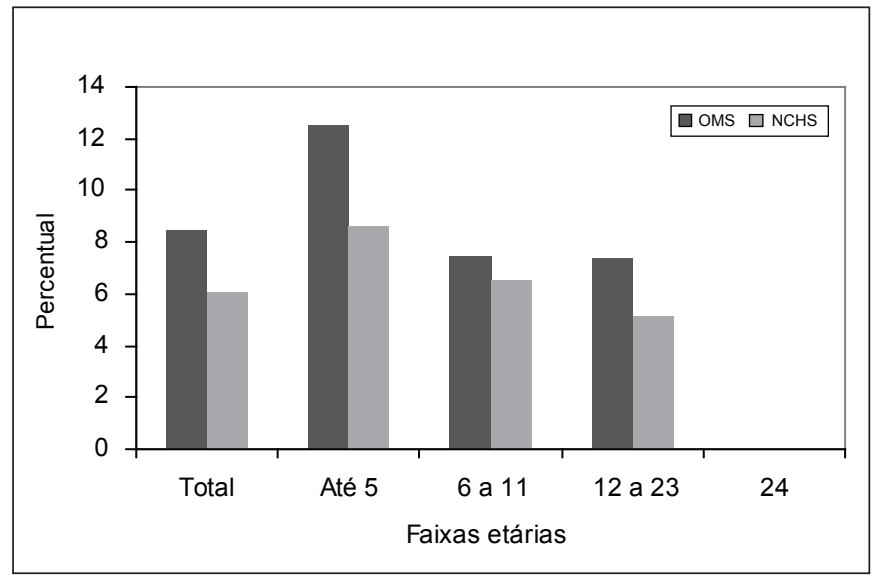

Figura 4 - Percentual de sobrepeso: curvas OMS e NCHS.

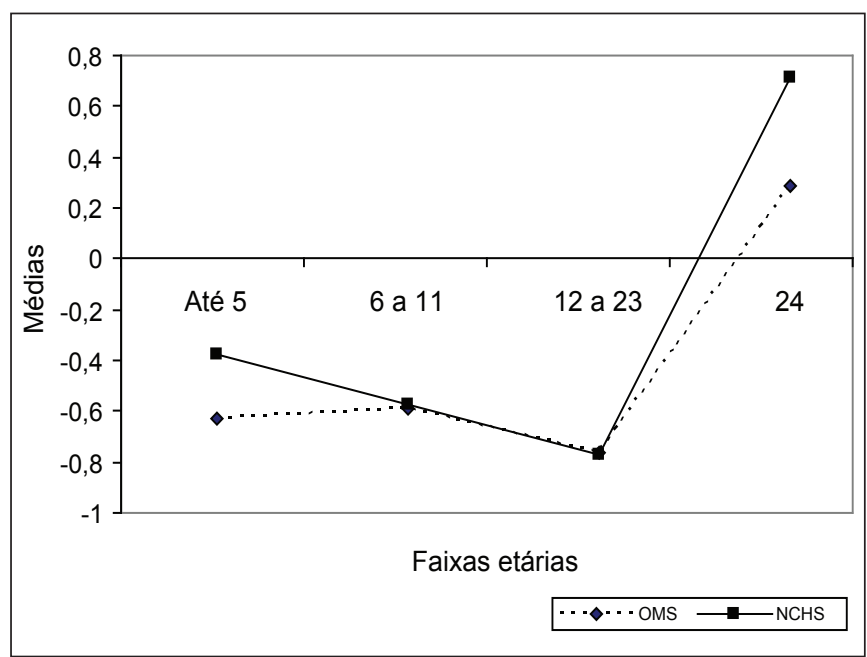

Figura 6 - Comparação das médias de altura/idade: curvas da OMS e NCHS. 
da família e que, se adequados, levariam a um padrão semelhante na evolução dos dados antropométricos. As diferenças raciais teriam, portanto, influência menor ${ }^{(10)}$, minimizando as prováveis interferências de diferenças étnicas na avaliação, mesmo considerando-se que apenas crianças norte-americanas foram analisadas na construção das curvas.

No entanto, alguns questionamentos já foram apontados por vários autores em relação à metodologia utilizada para a construção das curvas e, portanto, à sua utilização como padrão ideal. Entre esses, enfatiza-se o fato de que as populações estudadas eram constituídas predominantemente por crianças não amamentadas. A inclusão de crianças com hábitos alimentares considerados mais adequados por si só já justificaria a necessidade de novos padrões de crescimento. Além disso, os dados de crianças abaixo de três anos de idade eram originários de levantamentos feitos entre 1929 e 1975, considerados não representativos da população infantil atual ${ }^{(4-6)}$.

Assim, a OMS desenvolveu uma nova curva de referência, incluindo crianças amamentadas e que tiveram fatores ambientais favoráveis ao seu desenvolvimento. Nesse sentido, as novas curvas constituem um avanço, já que descrevem o crescimento de crianças que se utilizam, da melhor forma possível, de seu potencial. Portanto, podem ser consideradas não apenas descritivas, mas também prescritivas, já que representam a forma como as crianças "deveriam crescer" e não como crescem em um momento e local específicos ${ }^{(11)}$. Publicadas em 2006, as curvas podem ser acessadas em www.who.int/childgrowth/en. Trata-se de um documento extenso, com várias informações, gráficos e tabelas, que pode ser utilizado na prática clínica ${ }^{(12)}$.

As curvas do NCHS foram feitas com base em estudos com crianças sem os mesmos critérios de inclusão e exclusão definidos para a construção das curvas da OMS e que não foram amamentadas. Portanto, esperam-se diferenças significativas entre as duas referências. Neste estudo com crianças do Alto Jequitinhonha, foram observadas diferenças em todos os indicadores analisados. As curvas da OMS tiveram maior sensibilidade para a detecção de déficit peso/altura (wasting), exceto em crianças maiores, o mesmo acontecendo em relação ao déficit altura/idade (stunting), com prevalência maior quando se utilizou a curva da OMS. Já o déficit peso/idade (underweight) foi mais prevalente com a utilização das curvas da OMS apenas em crianças menores de seis meses de idade, sendo, a partir de então, observado um índice maior com a referência do NCHS. A detecção de sobrepeso também foi maior quando se utilizou a referência da OMS, em todas as faixas etárias estudadas. Esses resultados foram semelhantes aos encontrados em outro estudo ${ }^{(13)}$.

$\mathrm{Na}$ comparação entre as médias dos valores de escore $Z$, o peso apresenta valores maiores ao se utilizar a referência do NCHS até aproximadamente seis meses de idade, acontecendo o contrário posteriormente. Deve-se considerar a baixa duração do aleitamento materno exclusivo na população estudada, o que pode ter influenciado os resultados nos primeiros meses de vida. Os valores medianos de aleitamento materno exclusivo e aleitamento exclusivo foram 1,5 e 10,9 meses respectivamente ${ }^{(14,15)}$. Além disso, crianças com alimentação adequada devem crescer saudáveis, sem excesso de peso e tecido gorduroso e o sobrepeso é mais detectado pelas curvas da OMS, o que pode justificar as médias maiores de peso nesse caso. As médias de altura também são maiores nos primeiros meses com as curvas do NCHS e ocorrem ainda em crianças com 12 meses ou mais. Esses achados demonstram também um crescimento mais adequado nas crianças estudadas para a construção das curvas da OMS, as quais são mais sensíveis para a detecção de deficiências de crescimento.

É importante salientar que as crianças que participaram deste estudo são de famílias de baixo nível socioeconômico e residem em uma região pobre. A detecção mais frequente de deficiências nutricionais, principalmente crônicas, pode ser uma evidência de que as novas curvas são mais apropriadas, já que refletem uma situação ruim em relação às condições de vida.

As novas curvas da OMS foram testadas em outro estudo ${ }^{(2)}$, em crianças de países heterogêneos em relação à situação socioeconômica. Deficiências nutricionais foram mais encontradas no Paquistão, enquanto na Itália e Argentina as prevalências foram menores. Além disso, houve boa associação entre o diagnóstico clínico e a avaliação antropométrica com a utilização das novas curvas.

As curvas de crescimento da OMS constituem um instrumento mais adequado para avaliação de crianças, já que demonstram o crescimento quando as condições necessárias estão presentes. Neste estudo, foram observadas diferenças importantes quando as crianças foram avaliadas a partir das duas curvas. Considerando-se que vários estudos já verificaram que fatores ambientais são decisivos para um crescimento saudável, as novas curvas precisam ser implantadas e divulgadas, sendo enfatizada, junto à população e aos profissionais de saúde, a importância de uma alimentação adequada e dos cuidados básicos para o desenvolvimento de todas as crianças com a utilização de todo o seu potencial. 


\section{Referências bibliográficas}

1. Hamill PV, Drizd TA, Johnson CL, Reed RB, Roche AF. NCHS growth curves for children birth-18 years: United States. Vital Health Stat 1977;165:1-74.

2. Marcondes E, Berquó ES, Luongo J, Yunes J, Martins JS, Zacchi MA et al. Estudo antropométrico de crianças brasileiras de zero a doze anos de idade. São Paulo: Anais Nestlé; 1969.

3. Kuczmarski RJ, Ogden CL, Guo SS, Grummer-Strawn LM, Flegal KM, Mei Z et al. 2000 CDC growth charts for the United States: methods and development. Vital Health Stat 2002;246:1-190.

4. Araújo CL, Albernaz E, Tomasi E, Victora CG. Implementation of the WHO Multicentre Growth Reference Study in Brazil. Food Nutr Bul 2004;25:S53-8.

5. Onyango AW, Onis M, Caroli M, Shah U, Sguassero Y, Redondo N et al. Fieldtesting the WHO child growth standards in four countries. J Nutr 2007;137: 149-52.

6. Dibley MJ, Staehling N, Nieburg P, Trowbridge FL. Interpretation of Z-score anthropometric indicators derived from the international growth reference. Am J Clin Nutr 1987;46:749-62.

7. No authors listed. An evaluation of infant growth: the use and interpretation of anthropometry in infants. WHO Working Group on Infant Growth. Bul World Health Organ 1995;73:165-74.

8. Roberts SB, Dallal GE. The new childhood growth charts. Nutr Rev 2001;59:31-6.
9. Soares NT. A new growth anthropometric reference: meanings and implications. Rev Nutr 2003;16:93-104.

10. Onis M, Habicht JP. Anthropometric reference data for international use: recommendations from a World Health Organization Expert Committee. Am J Clin Nutr 1996;64:650-8.

11. Onis M, Victora CG. Growth charts for breastfed babies. J Pediatr (Rio J) 2004;80:85-7.

12. Organização Mundial da Saúde. The WHO child growth standards. [cited 2009 Apr 16]. Disponivel em: http://www.who.int/childgrowth/en.

13. Onis M, Onyango AW, Borghi E, Garza C, Yang H, WHO Multicentre Growth Reference Study Group. Comparison of the World Health Organization (WHO) child growth standards and the National Center for Health Statistics/WHO international growth reference: implications for child health programmes. Public Health Nutr 2006;9:942-7.

14. Silveira FJ, Lamounier JA. Breastfeeding prevalence and complementary feeding practices in children up to 24 months old in Alto Jequitinhonha region, Minas Gerais, Brazil. Rev Nutr 2004;17:437-47.

15. Silveira FJ, Lamounier JA. Factors associated with breastfeeding duration in three cities in the region of Alto Jequitinhonha, Minas Gerais, Brazil. Cad Saude Publica 2006;22:69-77. 